\title{
Flexible Bandwidth Allocation in Filterless Optical Networks
}

\author{
Zhenyu Xu, Christine Tremblay, Member, IEEE, Émile Archambault, Marija Furdek, Member, IEEE, \\ Jiajia Chen, Member, IEEE, Lena Wosinska, Senior Member, IEEE, Michel P. Bélanger, and Paul Littlewood
}

\begin{abstract}
We introduce the new concept of an elastic filterless optical network and propose an efficient heuristic algorithm for solving the static routing and spectrum assignment problem. Our simulation results obtained for different network topologies under multi-period traffic show increasing bandwidth savings with the growth of traffic load compared to a fixed-grid scenario. We also show the benefits of periodical spectrum defragmentation.
\end{abstract}

Index Terms-Elastic optical networking, flex-grid, routing and spectrum assignment (RSA), WDM.

\section{INTRODUCTION}

$\mathbf{E}$ LASTIC optical networking [1], [2], also referred to as flexible, gridless, and flex-grid networking, has the ability to improve spectral efficiency and flexibility, as channel spacing is no longer restricted to a fixed International Telecommunication Union (ITU) defined frequency grid, and specific channel bandwidths can be assigned to the traffic demands depending on the capacity and distance requirements. The corresponding bandwidth assignment problem is referred to as routing and spectrum assignment (RSA). Key technology enablers of elastic optical networks include multicarrier-based bandwidth variable transponders and flexible spectrum selective switches [1], [2].

The filterless optical network concept has been proposed as a simple and cost-effective architecture to deliver network agility [3], [4]. This concept is based on providing the network agility and reconfigurability by using tunable transmitters and coherent receivers at the terminals, as in radio networks. Consequently, in filterless optical networks, the active switching and colored components used at intermediate nodes and ingress/egress nodes, respectively, are replaced by passive optical splitters and combiners, leading to a significant cost reduction [3]. Additionally, the passive gridless architecture of filterless networks makes them suitable for elastic optical networking, thus avoiding replacement of the switching and filtering devices at nodes (in contrast to the current active

Manuscript received July 22, 2014; revised November 4, 2014 and December 11, 2014. This work was supported by the Natural Sciences and Engineering Research Council of Canada under grant CRDPJ 435362-12 and by Ciena Corp..

$\mathrm{Z}$. $\mathrm{Xu}$ and C. Tremblay are with the Department of Electrical Engineering, École de technologie supérieure, 1100 rue Notre-Dame Ouest, Montréal, Québec, H3C 1K3, Canada (email: christine.tremblay@etsmtl.ca).

É. Archambault is a member of the staff of the Cégep régional de Lanaudière, 20, St-Charles Sud, Joliette, Québec, J6E 4T1, Canada.

M. Furdek, J. Chen and L. Wosinska are with the KTH Royal Institute of Technology, Electrum 229, 16440 Kista, Sweden (email: wosinska@kth.se).

M. P. Bélanger and P. Littlewood are with Ciena Corp., 3500 Carling Avenue, Ottawa, ON, K2H 8E9, Canada (email: mbelange@ciena.com). photonic networks). Therefore, the gridless operation can be achieved at almost no cost without having to deploy gridless wavelength selective switches, which are at present significantly more expensive than fixed-grid ones.

The existing studies of filterless network architecture have been considering fixed-grid scenarios, i.e., the standard ITU grid with 50/100 GHz channels spacing. In [5], a design tool based on genetic and Tabu search algorithms was proposed to solve the physical link interconnection and static routing and wavelength assignment (RWA) problem in filterless networks. The analysis therein shows that filterless networks are cost effective and that resource optimization allows for keeping wavelength utilization within reasonable limits. The dynamic RWA problem in filterless networks was solved in [6]. A control plane for such networks was proposed in [7], while the resilience against link and node failures was studied in [8].

In this paper, we introduce the new concept of an elastic filterless optical network, which combines the benefits of filterless architectures based on passive broadcast-and-select (B\&S) nodes with the spectral efficiency and flexibility of elastic networking. An efficient heuristic algorithm is proposed for static RSA in elastic filterless networks under a multiperiod traffic scenario. A comparison with fixed-grid filterless solutions indicates superior performance of the elastic filterless approach in terms of spectrum utilization. Moreover, simulation results show that additional bandwidth savings can be achieved by periodical spectrum defragmentation.

\section{Elastic Filterless Optical Networks Concept}

The physical layer architecture of elastic filterless networks is composed of a set of passive B\&S nodes interconnected by edge-disjoint fiber trees. The fiber trees, each denoting a set of interconnected optical fiber links, represent a solution to the fiber link interconnection problem obtained by a genetic algorithm based design tool from [5], combined with the optical-layer protection scheme from [8] to provide $1+1$ dedicated protection. The resulting physical topology and network connectivity are established by configuring the splitters and combiners at the nodes.

Each node in the proposed elastic filterless network is equipped with coherent transponders [9], in which the modulation format and the corresponding channel capacity can be selected between quadrature phase shift keying (QPSK) and 16 quadrature amplitude modulation (QAM) through software control. In this work, we consider dual-polarization (DP) 


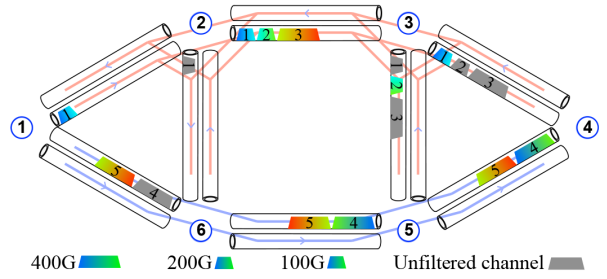

(a) Elastic filterless solution

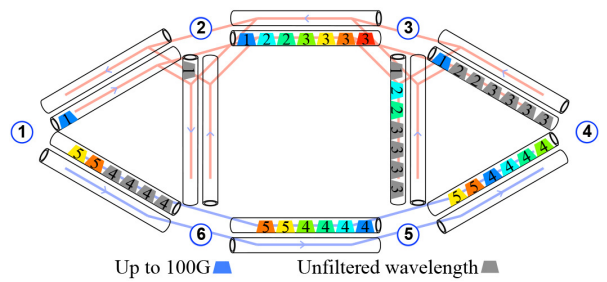

(b) Fixed-grid filterless solution

\begin{tabular}{|c|c|c|c|c|c|c|c|c|}
\hline Demand & Source & Destination & Traffic & Distance & Line rate & Modulation & BW (Flex) & BW (Fixed) \\
\hline 1 & 1 & 4 & $80 \mathrm{~Gb} / \mathrm{s}$ & $1200 \mathrm{~km}$ & $1 \times 100 \mathrm{G}$ & DP-QPSK & $3 \times 12.5 \mathrm{GHz}$ & $1 \times 50 \mathrm{GHz}$ \\
\hline 2 & 2 & 5 & $150 \mathrm{~Gb} / \mathrm{s}$ & $700 \mathrm{~km}$ & $1 \times 200 \mathrm{G}$ & DP-16QAM & $3 \times 12.5 \mathrm{GHz}$ & $2 \times 50 \mathrm{GHz}$ \\
\hline 3 & 2 & 3 & $380 \mathrm{~Gb} / \mathrm{s}$ & $300 \mathrm{~km}$ & $1 \times 400 \mathrm{G}$ & DP-16QAM & $6 \times 12.5 \mathrm{GHz}$ & $4 \times 50 \mathrm{GHz}$ \\
\hline 4 & 4 & 6 & $400 \mathrm{~Gb} / \mathrm{s}$ & $700 \mathrm{~km}$ & $2 \times 200 \mathrm{G}$ & DP-16QAM & $6 \times 12.5 \mathrm{GHz}$ & $4 \times 50 \mathrm{GHz}$ \\
\hline 5 & 4 & 1 & $200 \mathrm{~Gb} / \mathrm{s}$ & $1200 \mathrm{~km}$ & $2 \times 100 \mathrm{G}$ & DP-QPSK & $6 \times 12.5 \mathrm{GHz}$ & $2 \times 50 \mathrm{GHz}$ \\
\hline
\end{tabular}

(c) Traffic matrix

Figure 1. Illustration of elastic (a) and fixed-grid (b) filterless solutions in a six-node network topology for a given traffic matrix (c). The filterless solution with 2 fiber trees (blue and red, respectively) is shown in the background.

coherent transponders that can operate at channel capacities of 100 and $200 \mathrm{~Gb} / \mathrm{s}$ (single-carrier) and 400 (dual-carrier) Gb/s, with a corresponding channel bandwidth of $37.5 / 37.5 / 75 \mathrm{GHz}$ and reach of 2000/700/500 km, respectively [1], [10]. Softdecision forward error correction (SD-FEC) with overhead close to $20 \%$ is assumed in order to achieve the maximum reach at the considered line rates. On the other hand, 50-GHz channels at $100 \mathrm{~Gb} / \mathrm{s}$ are used in the fixed-grid case.

An illustration of the elastic filterless optical network concept is depicted in Fig 1 a Unlike the conventional $50-\mathrm{GHz}$ fixed-grid filterless solution shown in Fig $1 \mathrm{~b}$, the spectrum granularity of the elastic solution can be decreased to a single frequency slot unit (FSU) [1] (e.g., $12.5 \mathrm{GHz}$, used in Fig 1c. In principle, filterless optical networks do not have any limitation on the minimum FSU size, while the active switching based optical networks are typically restricted to a minimum FSU size (for example 6.25 or $12.5 \mathrm{GHz}$ ) due to the cumulative filtering at intermediate nodes.

The advantage of spectral efficiency in the elastic filterless network, compared to the fixed-grid case, is illustrated in Fig 1 through a spectrum allocation example. In general, spectrum assignment in elastic optical networks is subject to four constraints. Firstly, each traffic demand must be carried by the necessary number of consecutive FSUs throughout its physical route, which is referred to as the spectrum contiguity constraint. Secondly, each traffic demand must be assigned the same slots on all links it traverses, which is referred to as the spectrum continuity constraint. Thirdly, a guard band of $1 \mathrm{FSU}$ is needed between any two neighboring channels to mitigate the interference and crosstalk effects, referred to as the guard band constraint. Finally, there must be no spectrum overlapping between different connections. Thus, in the example from Fig 1 , the bandwidth (BW) required to carry

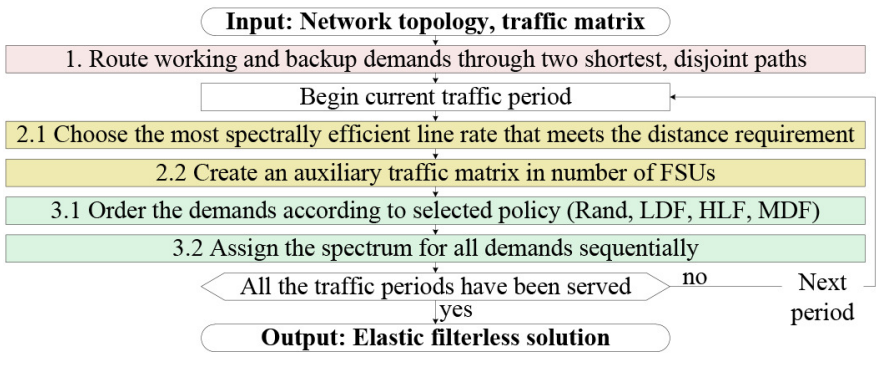

Figure 2. Flowchart of the proposed RSA-EF algorithm.

demands $1-3$ on the fiber link from node 2 to 3 is $175 \mathrm{GHz}$ (14 FSUs including two FSU guard bands between the three neighbouring channels) in the elastic filterless solution, while it requires $350 \mathrm{GHz}$ (seven $50-\mathrm{GHz}$ channels) in the fixed-grid filterless solution.

A major concern regarding resource consumption in filterless optical networks is the existence of unfiltered channels (shown in gray color in Fig $1 \mathrm{a}$ and $1 \mathrm{~b}$, which propagate all the way from the source to terminal nodes within a filterless fiber tree due to the drop-and-continue feature of filterless nodes [7]. The unfiltered channels increase the spectrum consumption, as the spectral resources occupied by them cannot be reused for other connections. Therefore, developing an efficient RSA algorithm is crucial to reduce the impact of unfiltered channels on the spectrum consumption.

\section{Problem Definition and Proposed RSA Algorithm FOR Elastic Filterless Networks} (RSA-EF)

The RSA problem in elastic filterless networks can be defined as follows. Given a traffic matrix for a certain time period and a physical network topology, where each node is equipped with coherent transponders supporting a set of line rates $L$ with specific spectrum width $B$, modulation format $M$, and reach $R$. The nodes are interconnected with a set of fiber trees ensuring $1+1$ dedicated path protection, generated using the methods from [5] and [8], as described above. To solve the RSA problem, we must decide on the working and the backup path for each connection request, select the most spectrallyefficient line rate whose reach is greater or equal to the path length, and assign spectral resources, i.e., FSUs. The objective is to optimize the overall spectrum utilization by minimizing the maximum number of FSUs used in each fiber link, while taking into account the constraints on the spectrum continuity, contiguity, overlapping and guard bands.

The flowchart of the proposed static RSA algorithm for elastic filterless networks, denoted as RSA-EF, is shown in Fig 2 Due to the NP-completeness of the RSA problem [11], it is solved in two sequential steps, i.e., routing (R) and spectrum assignment (SA). The routing sub-problem is solved by selecting a shortest pair of disjoint paths in the fiber tree topology for the working and the backup path of each traffic demand. When solving the SA sub-problem, we consider a multi-period traffic scenario modeling the long-term traffic growth. The SA sub-problem is solved for the incremental traffic in each period without re-assignment of the previously assigned spectrum. In the beginning of each traffic period, an 
TABLE I

RESULTS OF THE RSA ALGORITHM FOR SIX ELASTIC FILTERLESS OPTICAL NETWORK SOLUTIONS

\begin{tabular}{|c|c|c|c|c|c|c|c|c|c|c|c|}
\hline \multirow{3}{*}{ Network } & \multirow{3}{*}{ Topology } & \multirow{3}{*}{$\begin{array}{c}\text { Filterless } \\
\text { network } \\
\text { connectivity }\end{array}$} & \multirow{3}{*}{$\begin{array}{l}\text { Avg. demand } \\
\text { length }(\mathrm{km})\end{array}$} & \multirow{3}{*}{$\begin{array}{l}\text { Total } \\
\text { traffic } \\
(\mathrm{Tb} / \mathrm{s}) \\
\end{array}$} & \multicolumn{5}{|c|}{ Spectrum utilization (in number of FSUs) } & \multirow{3}{*}{$\begin{array}{l}\text { Spectrum } \\
\text { utilization } \\
\text { improvement }\end{array}$} & \multirow{3}{*}{$\begin{array}{c}\text { Percentage of } \\
\text { unfiltered spectrum } \\
\text { Elastic (Fixed-grid) }\end{array}$} \\
\hline & & & & & \multirow{2}{*}{ Fixed-grid } & \multicolumn{4}{|c|}{ Elastic } & & \\
\hline & & & & & & Rand & LDF & HLF & MDF & & \\
\hline \multirow{5}{*}{$\begin{array}{l}\text { 7-node } \\
\text { German }\end{array}$} & & \multirow{5}{*}{1.33} & \multirow{5}{*}{597} & 5.4 & 192 & 167 & 167 & 164 & 160 & $17 \%$ & \multirow{5}{*}{$40 \%(46 \%)$} \\
\hline & & & & 16.2 & 452 & 337 & 339 & 325 & 319 & $29 \%$ & \\
\hline & & & & 32.8 & 856 & 607 & 600 & 601 & 586 & $32 \%$ & \\
\hline & & & & 55.0 & 1408 & 977 & 963 & 947 & 956 & $33 \%$ & \\
\hline & & & & 82.8 & 2088 & 1401 & 1371 & 1367 & 1349 & $35 \%$ & \\
\hline \multirow{5}{*}{$\begin{array}{l}\text { 10-node } \\
\text { Italian }\end{array}$} & & \multirow{5}{*}{0.8} & \multirow{5}{*}{717} & 5.3 & 196 & 195 & 186 & 194 & 192 & $5 \%$ & \multirow{5}{*}{$32 \%(39 \%)$} \\
\hline & & & & 16.3 & 476 & 396 & 373 & 383 & 376 & $22 \%$ & \\
\hline & & & & 32.7 & 840 & 632 & 613 & 616 & 606 & $28 \%$ & \\
\hline & & & & 54.1 & 1312 & 956 & 912 & 949 & 923 & $30 \%$ & \\
\hline & & & & 81.8 & 1904 & 1346 & 1313 & 1336 & 1307 & $31 \%$ & \\
\hline \multirow{3}{*}{$\begin{array}{l}\text { Reference } \\
\text { network } 1\end{array}$} & & \multirow{3}{*}{0.48} & \multirow{3}{*}{836} & 7.8 & 192 & 222 & 220 & 215 & 210 & $-9 \%$ & \multirow{3}{*}{$25 \%(32 \%)$} \\
\hline & & & & 19.7 & 504 & 423 & 391 & 398 & 383 & $24 \%$ & \\
\hline & & & & 56.7 & 1288 & 975 & 912 & 914 & 894 & $31 \%$ & \\
\hline \multirow{3}{*}{$\begin{array}{l}\text { Reference } \\
\text { network } 2\end{array}$} & & \multirow{3}{*}{0.34} & \multirow{3}{*}{869} & 8.3 & 216 & 260 & 243 & 243 & 254 & $-13 \%$ & \multirow{3}{*}{$18 \%(23 \%)$} \\
\hline & & & & 21.4 & 540 & 472 & 450 & 454 & 454 & $17 \%$ & \\
\hline & & & & 63 & 1440 & 1089 & 1032 & 1017 & 1023 & $29 \%$ & \\
\hline \multirow{3}{*}{$\begin{array}{l}\text { Reference } \\
\text { network } 3\end{array}$} & & \multirow{3}{*}{0.37} & \multirow{3}{*}{785} & 6.8 & 160 & 198 & 197 & 200 & 196 & $-23 \%$ & \multirow{3}{*}{$19 \%(24 \%)$} \\
\hline & & & & 18.2 & 420 & 376 & 365 & 357 & 359 & $15 \%$ & \\
\hline & & & & 54.1 & 1092 & 849 & 809 & 800 & 789 & $28 \%$ & \\
\hline \multirow{3}{*}{$\begin{array}{l}\text { Reference } \\
\text { network } 4\end{array}$} & & & & 13.2 & 320 & 426 & 402 & 420 & 395 & $-23 \%$ & \\
\hline & & 0.24 & 809 & 35.8 & 872 & 811 & 770 & 782 & 755 & $13 \%$ & $23 \%(28 \%)$ \\
\hline & & & & 106.3 & 2324 & 1825 & 1757 & 1760 & 1705 & $27 \%$ & \\
\hline
\end{tabular}

auxiliary traffic matrix containing the extra requested FSUs per demand is generated to accommodate the incremental traffic. In order to minimize spectrum consumption, the most spectrally efficient line rate whose reach meets the demand path length is selected for each demand. The spectral efficiency is defined as $L /(B+G)$, where $G$ is the bandwidth of guard bands.

The algorithm proceeds by ordering the traffic demands for the current period using an ordering policy, and sequentially assigning FSUs to each demand. Three policies have been considered for ordering the demands: the longest distance first (LDF) (i.e., serve first the demand with the longest distance), the highest line rate first (HLF), and the most demanding first (MDF) (i.e., the largest value of the product of the path length and requested line rate). The algorithm first checks if a part of the traffic can be accommodated by the already used FSUs and modulation format between the same source-destination node pair. If the previously assigned bandwidth is not sufficient, the algorithm assigns the first available continuous spectrum from the short wavelength side to the current demand. The RSA-EF process is complete when all the demands in all periods have been accommodated.

In RSA-EF, the routing of both working and backup paths is predetermined for each demand before performing the SA, and a greedy algorithm is used to decide the ordering policy. The computational complexity of RSA-EF, for a single demand in a given period, depends linearly on the size of the fiber tree accommodating the demand because the algorithm needs to check every branch of the fiber tree due to the filterless B\&S node architecture. Therefore, the worst-case computational complexity in a single period can be expressed as $O(D \cdot N)$, where $D$ is the total number of demands and $N$ is the number of nodes in the network.

\section{Simulation Results}

The RSA-EF was implemented and its performance in terms of spectrum utilization was validated through simulations on the 7-node German network (G7), 10-node Italian network (IT10) [5], and 4 reference long haul network topologies (2000 km max. demand length), shown in Table I. Traffic matrices with incremental number of 100 random connections were used for the G7 and IT10 networks, while non-uniform traffic matrices representing realistic evolution scenarios for a time period of 5-10 years were used for the 4 reference networks. We analyzed the benefits of elastic versus fixed-grid deployment, as well as the influence of periodical spectrum defragmentation on the spectrum consumption.

In the elastic scenario, channel bandwidths $B$ equal to $37.5 / 37.5 / 75 \mathrm{GHz}$ with modulation formats $M$ of DPQPSK/DP-16QAM/DP-16QAM were assumed for line rates $L$ of $100 / 200 / 400 \mathrm{~Gb} / \mathrm{s}$, respectively, separated with guard bands $G=12.5 \mathrm{GHz}$ added at the end of each channel. We also assumed that up to 400 FSUs $(5 \mathrm{THz})$ are available on each fiber and that additional fibers can be deployed if the fiber capacity limit is reached. In addition to the three demand ordering policies considered in the SA sub-problem, a random ordering policy (Rand) has also been considered as a benchmark, and its results were averaged over 50 simulation instances for each network. The wavelength consumption for the fixed-grid scenario was obtained by applying the RWA algorithm from [5], where demands are accommodated using fixed 50-GHz wavelength channels of $100 \mathrm{~Gb} / \mathrm{s}$ capacity.

\section{A. Elastic vs. Fixed-grid Filterless Solutions}

The simulation results are summarized in Table I. The spectrum utilization improvement of the elastic solutions increases with the traffic load, as a result of always using channels with the highest spectral efficiency for demands shorter than $700 \mathrm{~km}$. The elastic solutions exhibit a significant reduction of spectrum utilization (from 27 to $35 \%$, depending on the network topology) at high traffic load, compared to the fixedgrid cases. However, the spectrum consumption advantage of elastic over fixed-grid reduces and even vanishes at low 


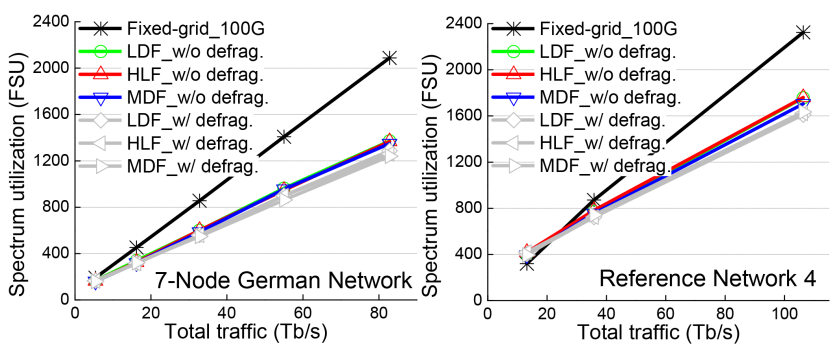

Figure 3. Spectrum utilization as a function of traffic load for two networks and two cases: "with defragmentation" vs. "without defragmentation".

traffic levels, as a consequence of always favoring the line rate with the most spectrally-efficient modulation format, leaving unused capacity for future usage.

Table I also shows the benefit of elastic spectrum assignment compared to the fixed-grid in filterless optical networks in terms of reduction of unfiltered spectrum, defined here as the ratio of the spectrum occupied by the unfiltered channels to the total utilized spectrum. As shown in the table, the percentage of unfiltered spectrum varies from 18 to $40 \%$, depending on the network topology, in the elastic filterless solutions, compared to 23 to $46 \%$ in the fixed-grid filterless solutions. As can be expected, the percentage of unfiltered spectrum increases with the filterless network connectivity, defined in $|8|$ as $2 k /(n(n-1))$, where $k$ is equal to the number of fiber link interconnections and $n$ to the number of nodes. On the other hand, the benefits of the proposed RSA-EF are particularly visible in the networks with relatively high connectivity. Additionally, the proposed demand ordering policies exhibit a moderate impact on spectrum utilization when compared to the random one.

\section{B. Impact of Periodical Spectrum Defragmentation}

We also evaluated the impact of spectrum defragmentation on the spectrum utilization for the elastic case (i.e., the algorithm starts at step 2.1 instead of 1 in Fig 2) for the G7 and reference 4 networks. In the first scenario, referred to as the "without defragmentation" case, the spectrum assigned in a certain traffic period does not get re-assigned in the following periods. The second scenario, referred to as the "with defragmentation" case, depicts an ideal situation in which all spectral resources can be reassigned in each traffic period. As can be seen from the results presented in Fig. 3, periodical spectrum defragmentation allows for additional improvements (from 6 to $10 \%$ ) in spectrum utilization. The benefits of spectrum defragmentation are moderate in the studied cases because the optimization process in the "without defragmentation" case aims at minimizing the spectrum consumption over long-term traffic evolution. It does so by selecting the most spectrally efficient line rate for each demand already in the starting period, leaving sufficient capacity free to accommodate the traffic growth in the subsequent periods without occupying additional spectrum.

\section{CONCLUSiON}

In this paper, we have introduced the new concept of an elastic filterless optical network and defined the static RSA prob- lem in such networks. We have proposed a tailored efficient RSA-EF algorithm for minimizing the spectrum consumption and tested it on 6 different network topologies under a multiperiod traffic evolution scenario. Simulation results show a reduction in spectrum utilization (of up to 35\%) at high traffic load, as well as in the percentage of unfiltered spectrum (of up to $6 \%$ ), compared with the fixed-grid case. The improvement in spectrum utilization is the most remarkable in the networks with higher connectivity, although the percentage of unfiltered spectrum therein is the most significant. Moreover, moderate improvements in spectrum consumption of elastic filterless optical networks have been obtained through periodical spectrum defragmentation. Although the cost factor is out of the scope of the study, the spectrum utilization improvement of elastic filterless solutions can also be expected to be delivered with a moderate cost impact, given that the difference between fixed-rate and flexible transponders resides mainly in the functionality integrated in the digital signal processing (DSP) modules.

The advantages and performance of elastic filterless networks can be further enhanced by formulating the RSA problem in elastic filterless networks using integer linear programming (ILP) to obtain optimal solutions with respect to different optimization criteria, or by designing more sophisticated heuristic RSA algorithms targeting the optimization of both cost and spectrum consumption in elastic filterless networks.

\section{REFERENCES}

[1] O. Gerstel, M. Jinno, A. Lord and S. J. B. Yoo, "Elastic optical networking: a new dawn for the optical layer?," IEEE Commun. Mag., vol. 50, no. 2, pp. s12-s20, Feb. 2012.

[2] K. Roberts, D. Beckett, D. Boertjes, J. Berthold and C. Laperle, "100G and beyond with digital coherent signal processing," IEEE Commun. Mag., vol. 48, no. 7, pp. 62-69, Jul. 2010.

[3] C. Tremblay, F. Gagnon, B. Châtelain, É. Bernier and M. P. Bélanger, "Filterless optical networks: a unique and novel passive WAN network solution," in 12th OECC-16th IOOC, 2007, paper 12P-7, pp. 466-467.

[4] C. Tremblay, É. Archambault, M. P. Bélanger, J. P. Savoie, F. Gagnon and D. V. Plant, "Passive filterless core networks based on advanced modulation and electrical compensation technologies," Telecommun. Syst., vol. 54, no. 2, pp. 167-181, Jul. 2013.

[5] É. Archambault, D. O’Brien, C. Tremblay, F. Gagnon, M. P. Bélanger and É. Bernier, "Design and simulation of filterless optical networks: problem definition and performance evaluation," IEEE/OSA J. Opt. Commun. Netw., vol. 2, no. 8, pp. 496-501, Aug. 2010.

[6] G. Mantelet, A. Cassidy, C. Tremblay, D. V. Plant, P. Littlewood and M. P. Bélanger, "Establishment of dynamic lightpaths in filterless optical networks," IEEE/OSA J. Opt. Commun. Netw., vol. 5, no. 9, pp. 10571065, Sep. 2013

[7] G. Mantelet, C. Tremblay, D. V. Plant, P. Littlewood and M. P. Bélanger, "PCE-based centralized control plane for filterless networks," IEEE Commun. Mag., vol. 51, no. 5, pp. 128-135, May 2013.

[8] Z. Xu, É. Archambault, C. Tremblay, J. Chen, L. Wosinska, M. P. Bélanger and P. Littlewood, " $1+1$ dedicated optical-layer protection strategy for filterless optical networks," IEEE Commun. Lett., vol. 18, no. 1 , pp. $98-101$, Jan. 2014.

[9] S. Gringeri, N. Bitar, T. J. Xia, "Extending software defined network principles to include optical transport," IEEE Commun. Mag., vol. 51, no. 3, pp. 32-40, Mar. 2013.

[10] K. Roberts and C. Laperie, "Flexible transceivers," in Proc. 38th ECOC, 2012, paper We.3.A.3.

[11] K. Christodoulopoulos, I. Tomkos and E. A. Varvarigos, "Elastic bandwidth allocation in flexible OFDM-based optical networks," J. Lightw. Technol., vol. 29, no. 9, pp. 1354-1366, May 2011. 